\title{
STRUCTURE OF THE ELECTROSPHERES OF BARE STRANGE STARS
}

\author{
V. V. Usov, ${ }^{1}$ T. Harko, ${ }^{2}$ and K. S. $\mathrm{CHENG}^{2}$ \\ Received 2004 September 2; accepted 2004 October 27
}

\begin{abstract}
We consider a thin $\left(\sim 10^{2}-10^{3} \mathrm{fm}\right)$ layer of electrons (the electrosphere) at the quark surface of a bare strange star, taking into account the surface effects at the boundary with the vacuum. The quark surface holds the electron layer by an extremely strong electric field, generated in the electrosphere to prevent the electrons from escaping to infinity by counterbalancing the degeneracy and thermal pressure. Because of the surface tension and depletion of $s$ quarks a very thin (a few $\mathrm{fm}$ ) charged layer of quarks forms at the surface of the star. The formation of this layer modifies the structure of the electrosphere by significantly changing the electric field and the density of the electrons, in comparison with the case in which the surface effects are ignored. Some consequences of the modification of the electrosphere structure on the properties of strange stars are briefly discussed.
\end{abstract}

Subject headings: dense matter - elementary particles — stars: neutron

\section{INTRODUCTION}

Strange stars made entirely of strange quark matter (SQM) have long been proposed as an alternative to neutron stars (Alcock et al. 1986; Haensel et al. 1986; Glendenning 1996; Cheng et al. 1998; Weber 1999; Cheng \& Harko 2000; Alford \& Reddy 2003; Lugones \& Horvath 2003 and references therein). The possible existence of strange stars is a direct consequence of the conjecture that SQM, composed of deconfined $u, d$, and $s$ quarks, may be the absolute ground state of the strong interaction, i.e., it is absolutely stable with respect to ${ }^{56} \mathrm{Fe}$ (Bodmer 1971; Witten 1984; Farhi \& Jaffe 1984). If SQM is approximated as a noninteracting gas of quarks, chemical equilibrium with respect to the weak interaction, together with the relatively large mass of the $s$ quarks, imply that the $s$ quarks are less abundant than the other quarks. Hence, in SQM electrons are required to neutralize the electric charge of the quarks. The electron density at vanishing pressure is of the order of $\sim 10^{-4}$ of the quark density (Alcock et al. 1986; Kettner et al. 1995).

The electrons, being bounded to SQM by the electromagnetic interaction alone, are able to move freely across the SQM surface, but clearly they cannot move to infinity because of the bulk electrostatic attraction to the quarks. The distribution of electrons extends several hundred fermis above the SQM surface, and an enormous electric field $E \simeq 5 \times 10^{17} \mathrm{~V} \mathrm{~cm}^{-1}$ is generated in the surface layer to prevent the electrons from escaping to infinity, counterbalancing the degeneracy and thermal pressure (Alcock et al. 1986; Kettner et al. 1995; Hu \& Xu 2002).

The thin layer of electrons with a very strong electric field, which is called the "electrosphere," drastically affects the observational appearance of strange stars. First, the electrosphere of a hot strange star with a bare SQM surface may be responsible for its thermal emission in a wide range of the surface temperature $T_{S}$. The point is that the electric field at the electrosphere is a few tens times higher than the critical field $E_{\mathrm{cr}} \simeq$ $1.3 \times 10^{16} \mathrm{~V} \mathrm{~cm}^{-1}$ at which vacuum is unstable to creation of $e^{+} e^{-}$pairs (Schwinger 1951). Therefore, a hot strange star with

\footnotetext{
1 Department of Condensed Matter Physics, Weizmann Institute, Rehovot 76100, Israel; fnusov@wicc.weizmann.ac.il.

2 Department of Physics, University of Hong Kong, Pokfulam Road, Hong Kong SAR, China; hrspksc@hkucc.hku.hk, harko@hkucc.hku.hk.
}

a bare SQM surface may be a powerful source of $e^{+} e^{-}$pairs, which are created in the electrosphere and flow away from the star (Usov 1998, 2001a; Aksenov et al. 2003, 2004). Emission of $e^{+} e^{-}$pairs from the electrosphere dominates in the thermal emission of a bare strange star at $6 \times 10^{8} \mathrm{~K} \lesssim T_{S} \lesssim 5 \times 10^{10} \mathrm{~K}$, while below this temperature, $T_{S} \lesssim 6 \times 10^{8} \mathrm{~K}$, bremsstrahlung radiation of photons from electron-electron collisions in the electrosphere prevails (Jaikumar et al. 2004). Besides, the flux of photons generated in the surface layer of SQM via any mechanism (for example, quark-quark bremsstrahlung) may be strongly reduced in the process of propagation through the electrosphere (Cheng \& Harko 2003). Second, the surface electric field may be also responsible for existence of a crust of "normal" matter (ions and electrons) at the SQM surface of a strange star (Alcock et al. 1986). This field is directed outward, and the ions in the inner layer are supported against the gravitational attraction to the underlying strange star by the electric field.

Recently, it was argued that the properties of the electrospheres may be changed essentially because of the surface effects (Usov 2004). It is the purpose of the present paper to study the structure of the electrospheres by taking into account the surface effects in detail. The different QCD phases of SQM are also considered. The inclusion of the surface effects drastically modifies the structure of the electrosphere by modifying the surface electric fields and the number density of the electrons.

The remainder of the paper is organized as follows. In $\S 2$ we formulate the equations that describe the distributions of the electric fields and the density of the electrons is the electrosphere and the boundary conditions. In $\S 3$ we obtain the solutions of the equations describing the properties of the electrosphere and discuss their properties. Finally, in $\S 4$ we summarize our results and discuss some astrophysical applications.

\section{FORMULATION OF THE PROBLEM}

In the electrosphere, electrons are held to the SQM surface by an extremely strong electric field. The thickness of the electrosphere is much smaller than the stellar radius, $R \simeq 10^{6} \mathrm{~cm}$, and a plane-parallel approximation may be used to study its structure. In this approximation all values depend only on the coordinate $z$, where the axis $z$ is perpendicular to the SQM surface $(z=0)$ and directed outward. To find the distributions 
of electrons and electric fields in the vicinity of the SQM surface, we use a simple Thomas-Fermi model considered by Alcock et al. (1986) and take into account both the finite temperature effects and the surface tension of SQM as discussed by Kettner et al. (1995) and Usov (2004), respectively. In the present paper we use units so that $\hbar=c=k_{\mathrm{B}}=1$. In these units, $e$ is equal to $\alpha^{1 / 2}$.

\subsection{Electrostatic Equilibrium Equations}

The chemical equilibrium of the electrons in the electric field implies that the value $\mu_{\infty}=\mu_{e}-e V$ is constant, where $V$ is the electrostatic potential and $\mu_{e}$ is the electron's chemical potential. Since far outside the star both $V$ and $\mu_{e}$ tend to zero, it follows that $\mu_{\infty}=0$ and $\mu_{e}=e V$.

The number density of the electrons is connected with the electron's chemical potential by the expression (Kettner et al. 1995; Cheng \& Harko 2003)

$$
n_{e}=\frac{1}{3 \pi^{2}} \mu_{e}^{3}+\frac{1}{3} \mu_{e} T_{S}^{2}=\frac{e V}{3 \pi^{2}}\left(e^{2} V^{2}+\pi^{2} T_{S}^{2}\right)
$$

where $T_{S}$ is the temperature of the electron layer, which is assumed to be constant in the layer and taken to be equal to the surface temperature of SQM. This is a very reasonable assumption because the thickness of the electron layer is very small and the electron density is very high. Therefore, the electrons have to be nearly in thermodynamic equilibrium with the SQM surface.

In the main part of the electrosphere the energy of electrons is significantly higher than the mass $m_{e}$, and the ultrarelativistic approximation when the energy of electrons is $\sim p$ may be used. In this approximation the Poisson equation takes the form (Alcock et al. 1986; Kettner et al. 1995; Cheng \& Harko 2003)

$$
\begin{gathered}
\frac{d^{2} V}{d z^{2}}=\frac{4 \alpha}{3 \pi}\left[e^{2}\left(V^{3}-V_{q}^{3}\right)+\pi^{2} T_{S}^{2}\left(V-V_{q}\right)\right], \quad z<0, \\
\frac{d^{2} V}{d z^{2}}=\frac{4 \alpha}{3 \pi}\left(e^{2} V^{3}+\pi^{2} T_{S}^{2} V\right), \quad z>0,
\end{gathered}
$$

where $\alpha \simeq 1 / 137$ is the fine-structure constant and $\left(\alpha / 3 \pi^{2}\right)$ $\times\left(e^{2} V_{q}^{3}+\pi^{2} T_{S}^{2} V_{q}\right)$ is the quark charge density.

The boundary conditions for equations (2) and (3) are $V \rightarrow$ $V_{q}$ and $d V / d z \rightarrow 0$ as $z \rightarrow-\infty$, and $V \rightarrow 0$ and $d V / d z \rightarrow 0$ as $z \rightarrow+\infty$, respectively.

To solve the Poisson equation and to find the distributions of electric fields and electrons it is necessary to know the quark charge density. In the following we consider the quark charge density for different QCD phases of SQM, taking into account the surface effects.

\subsection{Quark Charge Density in Bulk for Different QCD Phases}

For SQM made of noninteracting quarks the electric charge of the quarks is positive. Since SQM in bulk has to be electroneutral, electrons are required to neutralize the electric charge of the quarks.

The chemical potential $\left(\tilde{\mu}_{e}\right)$ of these electrons at zero temperature is usually used to characterize the quark charge density, $\tilde{\mu}_{e}=e V_{q}$. Below, we use only the chemical potential of electrons for SQM in bulk, and the tilde sign is omitted.

It is noted above that in noninteracting SQM the quarks are electrically charged because $s$ quarks are more massive than $u$ and $d$ quarks. The mass of $s$ quarks is likely between 50 and $300 \mathrm{MeV}$. The most traditional estimate is $m_{s} \simeq 150 \mathrm{MeV}$. The

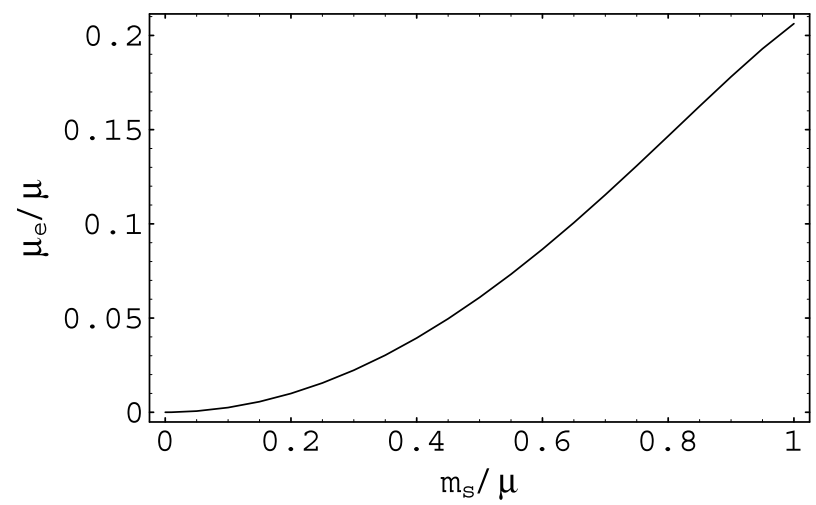

FIG. 1.-Electron chemical potential $\mu_{e}$ for unpaired SQM in bulk as a function of the $s$ quark mass $m_{s}$. Both these values are measured in the average quark chemical potential $\mu$.

masses of $u$ and $d$ quarks are less than $10 \mathrm{MeV}$, and we consider these light quarks as massless particles, $m_{u}=m_{d}=0$. Since $\mu_{e} \gg m_{e}$, we neglect the mass of electrons too, $m_{e}=0$.

Chemical equilibrium under weak reactions imposes

$$
\mu_{u}=\mu-\frac{2}{3} \mu_{e}, \quad \mu_{d}=\mu_{s}=\mu+\frac{1}{3} \mu_{e},
$$

where

$$
\mu=\frac{1}{3}\left(\mu_{u}+\mu_{d}+\mu_{s}\right)
$$

is the average quark chemical potential.

At low temperatures $\left(T \ll \mu_{e}\right)$, in thermodynamic equilibrium the number density of quarks are

$$
N_{u, d}=\frac{\mu_{u, d}^{3}}{\pi^{2}}, \quad N_{s}=\frac{\left(\mu_{s}^{2}-m_{s}^{2}\right)^{3 / 2}}{\pi^{2}}, \quad N_{e}=\frac{\mu_{e}^{3}}{3 \pi^{2}} .
$$

Electrical neutrality requires

$$
\frac{2}{3} N_{u}-\frac{1}{3} N_{d}-\frac{1}{3} N_{s}-N_{e}=0 .
$$

Equations (4)-(7) yield

$$
1-15 x+21 x^{2}-44 x^{3}-\left[(1+x)^{2}-y^{2}\right]^{3 / 2}=0,
$$

where $x=\mu_{e} / 3 \mu$ and $y=m_{s} / \mu$. Figure 1 shows a numerical solution of equation (8). This solution may be fitted by the expression

$$
\mu_{e}=0.248 \frac{m_{s}^{2}}{\mu}-0.007 \frac{m_{s}^{4}}{\mu^{3}}-0.034 \frac{m_{s}^{6}}{\mu^{5}},
$$

with the accuracy more than $1 \%$ for $m_{s} / \mu \leq 1$. The analytical estimate $\mu_{e} \simeq m_{s}^{2} / 4 \mu$ performed by Alford \& Rajagopal (2002) at $m_{s} / \mu \ll 1$ is well consistent with equation (9).

For strange stars, the value of $\mu$ is $\sim 300-350 \mathrm{MeV}$ at the surface and $\sim 400-500 \mathrm{MeV}$ at the center. Taking $m_{s} \simeq 150 \mathrm{MeV}$ and $\mu \simeq 300 \mathrm{MeV}$ as typical parameters of SQM at vanishing pressure, from equation (9) we have $\mu_{e} \simeq 18.3 \mathrm{MeV}$. This value of $\mu_{e}$ is usually used in consideration of the properties of noninteracting SQM. However, the range where $\mu_{e}$ may vary is very wide, from $\sim 2$ to $\sim 70 \mathrm{MeV}$.

It is becoming widely accepted that because of an attractive interaction between quarks in some specific channels, the ground state of SQM is a color superconductor (e.g., Bailin \& Love 1984; Alford et al. 1998, 2001a; Rapp et al. 1998; Evans et al. 
2000; Schäfer 2000; Alford 2001). At asymptotic densities $\left(\gg n_{0}\right)$, this superconductor is likely to be in the color-flavor locked (CFL) phase in which quarks of all three flavors and three colors are paired in a single condensate, where $n_{0} \simeq 0.16 \mathrm{fm}^{-3}$ is the normal nuclear density. Unfortunately, at the intermediate densities $\left(\sim 2 n_{0}\right)$ that are relevant to the SQM surface layers of strange stars, the QCD phase of SQM is uncertain. In this lowdensity regime, the SQM may be not only in the CFL phase but also in the "two-color-flavor superconductor" (2SC) phase in which only $u$ and $d$ quarks of second color are paired in a single condensate, while the ones of third color and the $s$ quarks of all three colors are unpaired. However, it was recently argued that the density range in which the 2SC phase may exist is small, if it exists at all (Alford \& Rajagopal 2002).

At rather low temperatures $(T \ll \Delta)$ the CFL phase in bulk is electrically neutral in the absence of any electrons, i.e., $\mu_{e}=$ $e V_{q}=0$, where $\Delta$ denotes the superconducting gap, $\Delta \sim 10$ $10^{2} \mathrm{MeV}$ (Rajagopal \& Wilczek 2001; Steiner et al. 2002; Weber 2004). The reason for the electrical neutrality is that BCSlike pairing minimizes the energy if the quark Fermi momenta are equal. In turn, for equal Fermi momenta the numbers of $u$, $d$, and $s$ quarks are equal and the electric charge of the quarks is zero. This differs qualitatively from the case of noninteracting SQM. At very high temperatures $(T \sim \Delta)$ the chemical potential of electrons in the CFL phase may be roughly estimated as $\sim \mu_{e} \exp (-\Delta / T)$, where $\mu_{e}$ is given by equation (9) (Weber 2004).

In the 2SC phase the chemical potential of electrons is, as rule, higher that the value given by equation (9) for noninteracting SQM, and it may be roughly estimated as $\mu_{e} \simeq \mu / 4$ (Huang \& Shovkovy 2003 and references therein). Numerically, for the 2SC phase in bulk at vanishing pressure we have $\mu_{e} \simeq 80 \mathrm{MeV}$.

In the CFL and 2SC phases, the Cooper pairs are made of quarks with equal and opposite momenta. Another possibility is a crystalline color superconductor (CCS), which involves pairing between quarks whose momenta do not add to zero (Alford et al. 2001b; Bowers \& Rajagopal 2002). Besides, recently it is shown that the gapless color-flavor locked (gCFL) and gapless two-color-flavor superconductor (g2SC) phases may also exist in addition to the regular CFL and 2SC phases (Shovkovy \& Huang 2003; Huang \& Shovkovy 2003; Alford et al. 2004). In the CCS, gCFL, and g2SC phases, electrons are present, and it is plausible that their chemical potential is also in the range from $\sim 10$ to $80 \mathrm{MeV}$.

\subsection{Thin Charged Layer at the Surface of SQM}

The density of quark states near the surface of SQM is modified and differs from the density of quark states in bulk (Berger \& Jaffe 1987; Berger 1991; Madsen 2001). This results in a sharp increase of quark charge density at the SQM surface. Indeed, the change in number of quarks of flavor $i$ per unit area because of surface tension is (Madsen 2000, 2001)

$$
n_{i, S}=-\frac{3}{4 \pi} p_{\mathrm{F}, i}^{2} \psi\left(\lambda_{i}\right),
$$

where

$$
\psi\left(\lambda_{i}\right)=\left[\frac{1}{2}+\frac{\lambda_{i}}{\pi}-\frac{1}{\pi}\left(1+\lambda_{i}^{2}\right) \tan ^{-1}\left(\lambda_{i}^{-1}\right)\right],
$$

$i=\{u, d, s\}, p_{\mathrm{F}, i}$ is the Fermi momentum of quarks of flavor $i, \lambda_{i}=m_{i} / p_{\mathrm{F}, i}$, and $m_{i}$ is the rest mass of quarks of flavor $i$. The value of $n_{i, S}$ is always negative, approaching zero for $\lambda_{i} \rightarrow 0$ (massless quarks).

The rest masses of $u$ and $d$ quarks are very small, and their densities are not modified significantly by the surface. Thus, the only appreciable contribution to the surface corrections arises from the $s$ quarks, i.e., surface effects are highly flavor dependent. Because of surface depletion of $s$ quarks, a thin charged layer forms at the surface of SQM. The charge per unit area is positive and equals

$$
\sigma=-\frac{1}{3} e n_{s, S} .
$$

The thickness of the charged layer at the SQM surface of SQM is of order of $1 \mathrm{fm}=10^{-13} \mathrm{~cm}$, which is a typical strong interaction length scale.

The thickness of the electron distribution in the electrosphere is about 2 order more than the thickness of the charged layer formed at the surface of SQM because of surface depletion of $s$ quarks (see Alcock et al. 1986; Kettner et al. 1995 and below), and therefore we assume that the last is infinitesimal. In this case, the $z$-component of the electric field

$$
E(z)=-d V / d z
$$

is discontinuous at the SQM surface $(z=0)$, and the electric field jump is

$$
\Delta E=E_{\text {ext }}(+0)-E_{\text {int }}(-0)=4 \pi \sigma
$$

where $E_{\mathrm{ext}}(+0)$ and $E_{\mathrm{int}}(-0)$ are the $z$-components of the electric field at the external $(z=+0)$ and internal $(z=-0)$ sides of the SQM surface, respectively.

For $m_{s} \simeq 150 \mathrm{MeV}$ and $p_{\mathrm{F}, s} \simeq 300 \mathrm{MeV}$, from equations (10)(14) we have $\Delta E \simeq 5.5 \times 10^{18} \mathrm{~V} \mathrm{~cm}^{-1} \simeq 4 \times 10^{2} E_{\mathrm{cr}}$, which is $\sim 10$ times larger than the surface electric field calculated for SQM in the unpaired phase when the surface effects are neglected (Alcock et al. 1986; Kettner et al. 1995; Hu \& Xu 2002). Therefore, the charged layer formed at the surface of SQM because of the surface depletion of $s$ quarks changes the structure of electrospheres essentially (see below).

The thermal effects have been neglected in the derivation of equation (10). However, since the thermal energy is small in comparison with the energy of quarks even if the temperature is as high as a few times $10 \mathrm{MeV}$, this approximation does not affect the conclusions of this paper.

\section{STRUCTURE OF ELECTROSPHERES}

In our study the thermal effects for electrons in the electrosphere are taken into account because a strange star at the moment of formation may have a surface temperature $T_{S}$ comparable with the Fermi energy of electrons inside SQM.

\subsection{Hot Electrospheres}

The first integrals of equations (2) and (3), which satisfy the boundary conditions at $z \rightarrow \pm \infty$, are

$$
\begin{aligned}
\frac{d V}{d z}= & \pm\left(\frac{2 \alpha}{3 \pi}\right)^{1 / 2} \\
& \times\left[e^{2}\left(V^{4}-4 V_{q}^{3} V+3 V_{q}^{4}\right)+2 \pi^{2} T_{S}^{2}\left(V-V_{q}\right)^{2}\right]^{1 / 2}
\end{aligned}
$$


and

$$
\frac{d V}{d z}= \pm\left(\frac{2 \alpha}{3 \pi}\right)^{1 / 2}\left(e^{2} V^{4}+2 \pi^{2} T_{S}^{2} V^{2}\right)^{1 / 2}
$$

at $z<0$ and $z>0$, respectively.

Outside of the SQM surface $(z>0)$, the $z$-component of the electric field is directed outward $(E>0)$ to prevent electrons of the electrosphere from escaping. Therefore, the minus sign has to be chosen in equation (16) and the external electric field is

$$
E_{\mathrm{ext}}=\left(\frac{2 \alpha}{3 \pi}\right)^{1 / 2}\left(e^{2} V^{4}+2 \pi^{2} T_{S}^{2} V^{2}\right)^{1 / 2} .
$$

This equation is valid for any numerical values of $\sigma$ and $V_{q}$. Integration of equations (13) and (17) yields the electrostatic potential at $z \geq 0$ (Cheng \& Harko 2003)

$$
V=\frac{2 \sqrt{2} \pi T_{S} \exp 2 \sqrt{(\pi \alpha / 3)} T_{S}\left(z+z_{0}\right)}{e\left\{\exp \left[4 \sqrt{(\pi \alpha / 3)} T_{S}\left(z+z_{0}\right)\right]-1\right\}},
$$

where

$$
z_{0}=\sqrt{\frac{3}{\pi \alpha}} \frac{1}{2 T_{S}} \ln \left(\frac{\sqrt{2} \pi T_{S}}{e V_{0}}+\sqrt{1+\frac{2 \pi^{2} T_{S}^{2}}{e^{2} V_{0}^{2}}}\right)
$$

is a constant of integration and $V_{0}$ is the electrostatic potential at the SQM surface $(z=0)$.

Equations (17)-(19) determine the external electric field as a function of $V_{0}$ and $T_{S}$. To find the value of $V_{0}$ it is necessary to consider the electric field inside the SQM surface $(z<0)$.

The direction of the internal electric field may be different depending on the values of $\sigma$ and $V_{q}$.

Here the following two cases are considered. In the first case, when $\sigma=0$ and $V_{q} \neq 0$, the internal electric field is directed outward, similar to the external electric field (Alcock et al. 1986; Kettner et al. 1995). In the second case, in which $\sigma \neq 0$ and $V_{q}=0$, the electric field is symmetric with respect to the SQM surface $[E(-z)=-E(z)]$, i.e., the internal electric field is directed inward (Usov 2004).

In the last case the electric field is discontinuous at the SQM surface, and its strength is equal to $\Delta E / 2=2 \pi \sigma$ at $z=0$.

Before considering the internal electric field in a general case in which $\sigma \neq 0$ and $V_{q} \neq 0$, we find a value of $\sigma=\sigma_{0}$ at which the internal electric field is zero for a given value of $V_{q}$.

From equations (13) and (15), we have a solution $E_{\text {int }}=0$ if $V=V_{q}$ at $z<0$. At the SQM surface the electrostatic potential $V$ is continuous, and therefore $V_{0}=V_{q}$ for this solution, while the electric field is discontinuous, $\Delta E=E_{\text {ext }}(+0)=4 \pi \sigma_{0}$. Substituting $E_{\text {ext }}(+0)$ from this equation into equation (17) where $V=V_{q}$ we get

$$
\sigma_{0}=\frac{1}{4 \pi}\left(\frac{2 \alpha}{3 \pi}\right)^{1 / 2}\left(e^{2} V_{q}^{4}+2 \pi^{2} T_{S}^{2} V_{q}^{2}\right)^{1 / 2}
$$

To characterize the importance of the surface effects for the generation of strong electric fields in the vicinity of the SQM surface we introduce the parameter

$$
\eta=\frac{\sigma}{\sigma_{0}}=\frac{\Delta E}{E_{q}},
$$

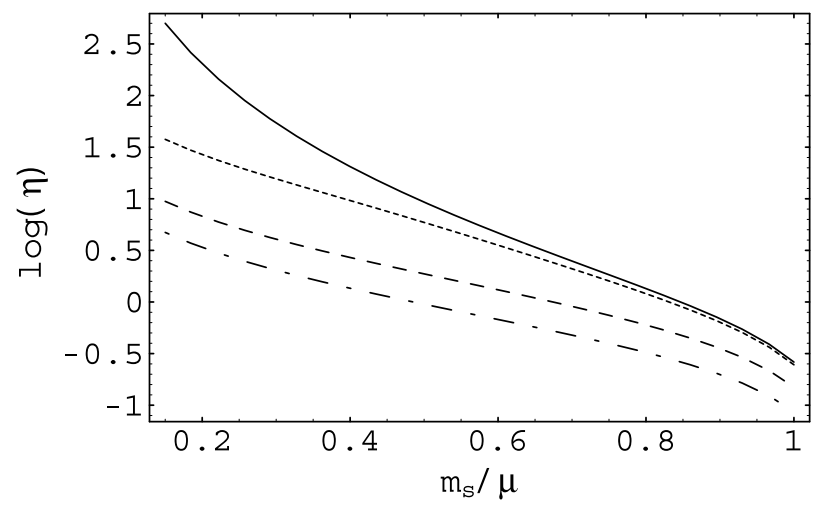

FIG. 2.-Parameter $\eta=\sigma / \sigma_{0}$ as a function of the ratio of the $s$ quark mass $m_{s}$ and the average quark chemical potential $\mu$ for different temperatures at the SQM surface: $T_{S}=0$ (solid curve), $T_{S}=5 \mathrm{MeV}$ (dotted curve), $T_{S}=20 \mathrm{MeV}$ (dashed curve), and $T_{S}=40 \mathrm{MeV}$ (dash-dotted curve).

where $E_{q}$ is the strength of the external electric field given by equation (17) at $V=V_{q}$. The surface effects are not essential for the electrosphere structure if the jump of the electric field $\Delta E$ is much smaller than the electric field near the SQM surface, i.e., if $\eta \ll 1$. At $\eta \geq 1$, the electric field structure in the vicinity of the SQM surface changes qualitatively in comparison with the field structure considered by Alcock et al. (1986) and Kettner et al. (1995) when the surface effects have been ignored $(\eta=0)$. For example, at $\eta>1$ the internal electric field is directed inward $\left(E_{\text {int }}<0\right)$, while at $\eta=0$ it is directed outward $\left(E_{\text {int }}>0\right)$. Besides, in the former case the electric field and the density of electrons in the electrosphere increase significantly (see below).

Equations (10), (12), (20), and (21) yield

$$
\eta=\eta_{0}\left(1+\frac{2 \pi^{2} T_{S}^{2}}{e^{2} V_{q}^{2}}\right)^{-1 / 2}
$$

where

$$
\eta_{0}=\left(\frac{3 \pi \alpha}{2}\right)^{1 / 2}\left(\frac{p_{\mathrm{F}, s}}{e V_{q}}\right)^{2} \psi\left(\lambda_{s}\right)
$$

For the most conservative parameters, $m_{s} \simeq 150 \mathrm{MeV}, p_{\mathrm{F}, s} \simeq$ $300 \mathrm{MeV}$, and $e V_{q} \simeq 20 \mathrm{MeV}$, from equations (22) and (23) we have $\eta_{0} \simeq 9$ and

$$
\eta \simeq 9\left[1+\left(\frac{T_{S}}{4.5 \mathrm{MeV}}\right)^{2}\right]^{-1 / 2}
$$

From this equation we can see that if the surface temperature is not extremely high $\left(T_{S}<40 \mathrm{MeV}\right), \eta$ is greater than 1, i.e., the surface depletion of $s$ quarks is mainly responsible for the generation of extremely strong electric fields near the SQM surface, not the electrons of SQM in bulk.

Using equations (4), (9), (11), (22), and (23), we performed numerical calculations of $\eta$ for unpaired SQM at different temperatures, $0 \leq T_{S} \leq 40 \mathrm{MeV}$. Figure 2 shows $\eta$ as a function of $m_{s} / \mu$ for $\mu=300 \mathrm{MeV}$ and $50 \mathrm{MeV} \leq m_{s} \leq 300 \mathrm{MeV}$. From Figure 2 we can see that $\eta$ is in the range from $\sim 3 \times 10^{2}$ at $m_{s} \simeq 50 \mathrm{MeV}$ and low temperatures $\left(T_{S} \lesssim \mathrm{a}\right.$ few $\left.\mathrm{MeV}\right)$ to $\sim 0.1$ at $m_{S} \simeq 300 \mathrm{MeV}$ and $T_{S} \simeq 40 \mathrm{MeV}$.

Hence, both cases $(\eta>1$ and $\eta<1)$ may occur in the electrospheres of bare strange stars, depending on the surface 


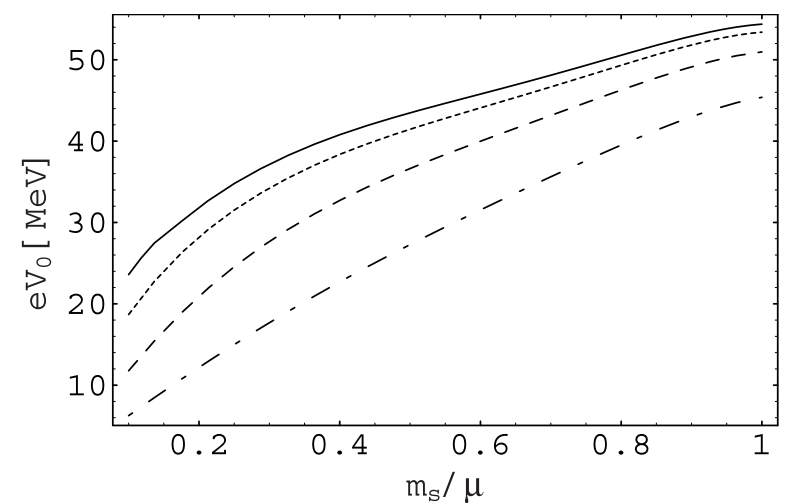

FIG. 3.-Electrostatic potential of electrons $e V_{0}$ at the surface of unpaired SQM as a function of the ratio of the $s$ quark mass $m_{s}$ and the average quark chemical potential $\mu$ for $\mu=300 \mathrm{MeV}$ and different temperatures at the SQM surface: $T_{S}=0$ (solid curve), $T_{S}=5 \mathrm{MeV}$ (dotted curve), $T_{S}=10 \mathrm{MeV}$ (dashed curve), and $T_{S}=20 \mathrm{MeV}$ (dash-dotted curve).

temperature and the SQM parameters. Since the internal electric field is directed outward at $\eta<1$ and inward at $\eta>1$, the sign - or + has to be taken in equation (15) at $\eta<1$ or $\eta>1$, respectively.

The sign of $1-\eta$ coincides with the sign of $V-V_{q}$ at $z \leq 0$, and from equations (13) and (15) the internal electric field may be written in the form

$$
\begin{aligned}
E_{\text {int }}= & -\frac{d V}{d z}=\left(\frac{2 \alpha}{3 \pi}\right)^{1 / 2}\left(V_{q}-V\right) \\
& \times\left[e^{2}\left(V+V_{q}\right)^{2}+2 e^{2} V_{q}^{2}+2 \pi^{2} T_{S}^{2}\right]^{1 / 2},
\end{aligned}
$$

which is valid for any value of $\eta$.

Integration of equation (25) yields the electrostatic potential at $z \leq 0$,

$$
V=\left[\frac{(1+\xi \operatorname{coth} \chi)^{2}-\xi^{2}+1}{1+\xi \operatorname{coth} \chi}-1\right] V_{q}
$$

where

$$
\begin{gathered}
\xi=\left[\frac{3}{2}\left(1+\frac{\pi^{2} T_{S}^{2}}{3 e^{2} V_{q}^{2}}\right)\right]^{1 / 2}, \\
\chi=\exp \left[\left(\frac{2 \alpha}{3 \pi}\right)^{1 / 2} e V_{q} \xi\left(|z|+\tilde{z}_{0}\right)\right], \\
\tilde{z}_{0}=\left(\frac{3 \pi}{2 \alpha}\right)^{1 / 2} \frac{1}{\xi e V_{q}} \ln \operatorname{coth}^{-1} \\
\times\left[\frac{1}{2 \xi}\left(\frac{V_{0}}{V_{q}}-1\right)+\frac{1}{2 \xi} \sqrt{\left(\frac{V_{0}}{V_{q}}+1\right)^{2}+4\left(\xi^{2}-1\right)}\right] .
\end{gathered}
$$

Substituting $\sigma, E_{\text {ext }}(+0)$, and $E_{\text {int }}(-0)$ from equations (12), (17), and (25) into equation (14), we have the following equation for the electrostatic potential $V_{0}$ at $z=0$ :

$$
\begin{aligned}
& \left(V_{0}-V_{q}\right)\left[e^{2}\left(V_{0}+V_{q}\right)^{2}+2 e^{2} V_{q}^{2}+2 \pi^{2} T_{S}^{2}\right]^{1 / 2} \\
& +\left(e^{2} V_{0}^{4}+2 \pi^{2} T_{S}^{2} V_{0}^{2}\right)^{1 / 2}=(3 \pi / 2)^{1 / 2} p_{\mathrm{F}, s}^{2} \psi\left(\lambda_{s}\right) .
\end{aligned}
$$

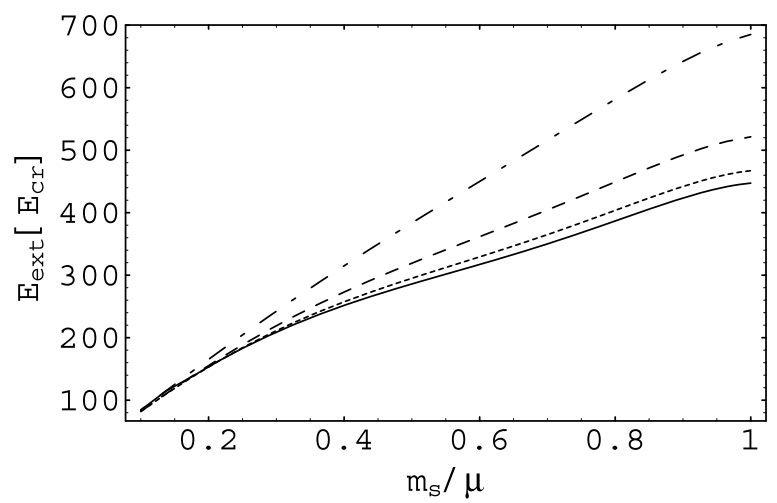

FIG. 4.-External electric field (in units of $E_{\mathrm{cr}}$ ) at the SQM surface as a function of the ratio of the mass of $s$ quarks and the average quark chemical potential $\mu$ for $\mu=300 \mathrm{MeV}$ and different temperatures at the SQM surface: $T_{S}=0$ (solid curve), $T_{S}=5 \mathrm{MeV}$ (dotted curve), $T_{S}=10 \mathrm{MeV}$ (dashed curve), and $T_{S}=20 \mathrm{MeV}$ (dash-dotted curve).

Figure 3 shows the value of $V_{0}$ at the surface of unpaired SQM as a function of $m_{s} / \mu$ for $\mu \simeq 300 \mathrm{MeV}$ and different values of $T_{S}$.

Equations (1), (17)-(19), and (25)-(30) determine the electric field and the density of electrons in the electrosphere of a bare strange star as functions of $m_{s}, \mu, V_{q}$, and $T_{S}$. In turn, $V_{q}$ is not a free parameter and may be calculated for different QCD phases of SQM as functions of $m_{s}, \mu$, and $T_{S}$ (see $\S 2$ ). From Figure 4 we see that the external field at the SQM surface is extremely high $\left[\sim(1-7) \times 10^{2} E_{\text {cr }}\right]$, which is $\sim 10$ times more than the same without taking into account the surface effects. Figures 5 and 6 show the profiles of the electrostatic potential of electrons and the electron density in the vicinity of the SQM surface, respectively.

\subsection{Cold Electrospheres}

At $\sim 10 \mathrm{~s}$ after the formation of a bare strange star the surface temperature is (Page \& Usov 2002)

$$
T_{S} \ll T_{*}=\frac{1}{\pi} e V_{0} \sim 10 \mathrm{MeV}
$$

From equations (1)-(3) it follows that in this case the thermal effects are not essential for the distributions of electrons and electric fields near the SQM surface. Even if the surface of a

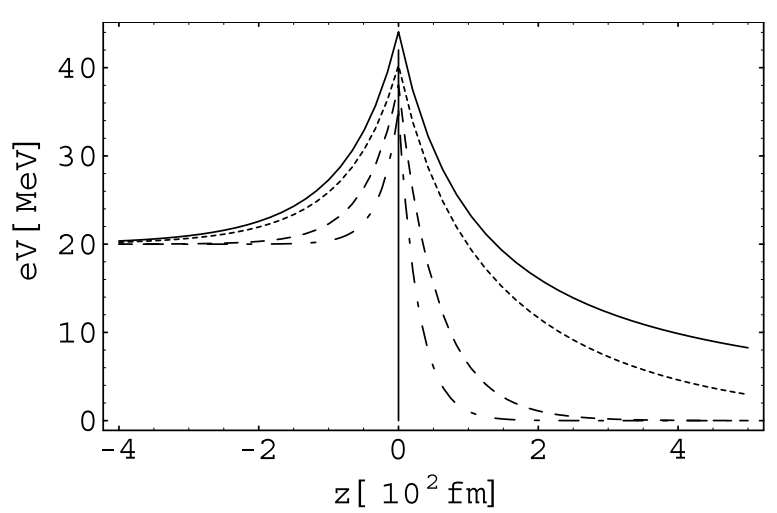

FIG. 5.-Electrostatic potential of electrons $\mathrm{eV}$ as a function of the distance $z$ from the SQM surface $(z=0)$ for $m_{s}=150 \mathrm{MeV}, \mu=300 \mathrm{MeV}, e V_{q}=20 \mathrm{MeV}$, and for different temperatures at the surface: $T_{S}=0$ (solid curve), $T_{S}=5 \mathrm{MeV}$ (dotted curve), $T_{S}=20 \mathrm{MeV}$ (dashed curve), and $T_{S}=40 \mathrm{MeV}$ (dash-dotted curve). 


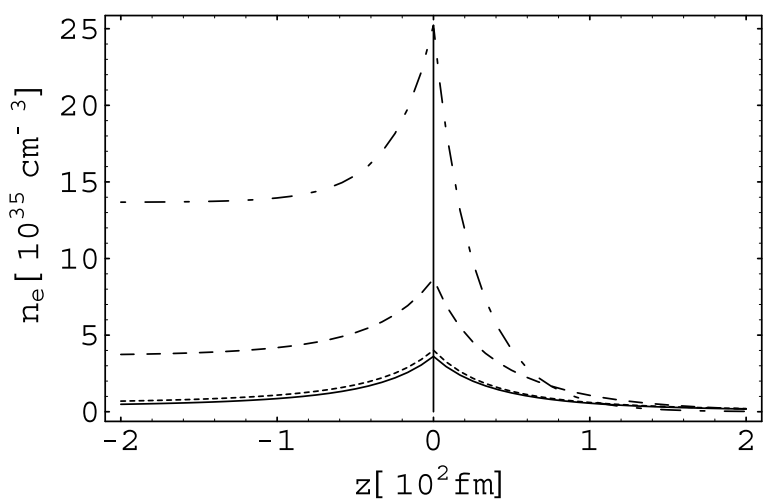

FIG. 6.-Electron number density profiles near the SQM surface for $m_{s}=$ $150 \mathrm{MeV}, \mu=300 \mathrm{MeV}, e V_{q}=20 \mathrm{MeV}$, and for different temperatures at the surface: $T_{S}=0$ (solid curve), $T_{S}=5 \mathrm{MeV}$ (dotted curve), $T_{S}=20 \mathrm{MeV}$ (dashed curve), and $T_{S}=40 \mathrm{MeV}$ (dash-dotted curve).

bare strange star is occasionally heated by accretion of matter, for example, so that the thermal luminosity of the star is as high as $\sim 10^{44}-10^{45} \mathrm{ergs} \mathrm{s}^{-1}$, the surface temperature is $T_{S} \simeq$ (1-2) $\times 10^{9} \mathrm{~K} \ll T_{*}$ (Usov 2001b). Hence, strange stars in the process of their evolution are mostly at the state in which $T_{S} \ll T_{*}$, and therefore we discuss this low-temperature case especially.

In this case the electrostatic potential at $z \geq 0$ has a very simple form:

$$
V=\left(\frac{3 \pi}{2 \alpha}\right)^{1 / 2} \frac{1}{e\left(z+z_{0}\right)}=\frac{C}{e\left(z+z_{0}\right)},
$$

where

$$
z_{0}=\left(\frac{3 \pi}{2 \alpha}\right)^{1 / 2} \frac{1}{e V_{0}}=\frac{C}{e V_{0}}
$$

is the typical thickness of the electrosphere and $C=$ $(3 \pi / 2 \alpha)^{1 / 2}=5.013 \times 10^{3} \mathrm{MeV}$ fm. For $e V_{0}=40 \mathrm{MeV}$, we have $z_{0} \simeq 125 \mathrm{fm}$.

From equations (1), (17), and (32), the external electric field and the density of electrons are, respectively,

$$
\begin{gathered}
E_{\mathrm{ext}}=\frac{C}{e\left(z+z_{0}\right)^{2}}, \\
n_{e}=\frac{1}{3 \pi^{2}}\left(\frac{3 \pi}{2 \alpha}\right)^{3 / 2} \frac{1}{\left(z+z_{0}\right)^{3}} .
\end{gathered}
$$

The electrostatic potential inside the SQM surface is not simplified significantly at low temperatures, and is given by equations $(26),(28)$, and (29) where $\xi=(3 / 2)^{1 / 2}$.

From equations (23) and (30), in the limit of zero temperature the equation for $V_{0}$ is

$$
\left(V_{0}-V_{q}\right)\left[\left(V_{0}+V_{q}\right)^{2}+2 V_{q}^{2}\right]^{1 / 2}+V_{0}^{2}=\eta_{0} V_{q}^{2}
$$

This equation has the solution

$$
V_{0}=\left[\frac{1}{\eta_{0}} \pm\left(\frac{1}{\eta_{0}^{2}}+\frac{\eta_{0}^{2}-3}{2 \eta_{0}}\right)^{1 / 2}\right] V_{q}
$$

where the sign + or - has to be taken at $\eta \geq 1$ or $\eta<0$, respectively.

For $\eta_{0} \simeq 9$, which relates to the most conservative parameters of SQM (see above), from equation (37) we have $V_{0} \simeq$ $2.2 V_{q}$, which is 2.93 times more than the $V_{0}=(3 / 4) V_{q}$ found in many papers (e.g., Alcock et al. 1986; Kettner et al. 1995) in which the surface effects were ignored. Then, from equations (1) and (17) taken at $T_{S}=0$, we find that at the SQM surface the external electric field $\left(E_{\text {ext }} \propto V_{0}^{2}\right)$ and the density of electrons $\left(n_{e} \propto\right.$ $V_{0}^{3}$ ) increase because of the surface effects by factors of 8.6 and 25 , respectively, and are $E_{\text {ext }}(z=0) \simeq 4 \times 10^{18} \mathrm{~V} \mathrm{~cm}^{-1} \simeq$ $3 \times 10^{2} E_{\mathrm{cr}}$ and $n_{e}(z=0) \simeq 3.7 \times 10^{35} \mathrm{~cm}^{-3}$.

\section{DISCUSSION}

In this paper, we have considered the structure of extremely strong electric fields and the distribution of the density of electrons in the vicinity of the SQM surface of a bare strange star.

We take into account that a very thin (a few fm) charged layer forms at the SQM surface because of the surface depletion of $s$ quarks. We have shown that the surface charged layer significantly changes both the field structure and the density of electrons as compared with the same calculated in many papers (e.g., Alcock et al. 1986; Kettner et al. 1995; Hu \& Xu 2002; Cheng \& Harko 2003) where the surface effects are ignored. These changes are especially large if the surface temperature is not very high $\left(T_{S} \lesssim\right.$ a few $\left.\mathrm{MeV}\right)$.

For a bare strange star, the structure of electric fields and the density of electrons near the stellar surface depend on $m_{s}, \mu$, and $T_{S}$ (see eqs. [1], [17]-[19], [25]-[30], and $\S 2$ ). For SQM at vanishing pressure, the value of $\mu$ is $\sim 300 \mathrm{MeV}$ with an accuracy of $\sim 20 \%$, and the main uncertainty of the parameters of the electrospheres of bare strange stars is because of uncertainty of $m_{s}$, which is estimated as $150 \mathrm{MeV}$ within a factor of $2-3$ or so.

The density of electrons in the electrosphere of a bare strange star increases because of the surface effects by a factor of a few times 10 ( see $\S 3$ ). This may significantly change the thermal emission from the stellar surface. In $\S 1$, the main mechanisms of the thermal emission are mentioned. These are the creation of $e^{+} e^{-}$pairs in a supercritical electric field (Usov 1998), quarkquark bremsstrahlung from the surface layer of SQM (Cheng \& Harko 2003), and electron-electron bremsstrahlung from the electron layer (Jaikumar et al. 2004). In supercritical electric fields, $E \gg E_{\mathrm{cr}}$, the rate of pair production when electrons are created into the empty quantum states is extremely high (Schwinger 1951), and all the empty states are occupied by creating electrons practically instantly. Then, the rate of pair production in the electrosphere is determined by the process of thermalization of electrons, which favors the empty-state production (Usov 1998). Using this, we have roughly the following dependence of the strange star luminosity in $e^{+} e^{-}$pairs on $V_{0}$ :

$$
L_{ \pm} \propto \begin{cases}\text { constant, } & T_{S}<0.01 e V_{0} \\ V_{0}^{1.8}, & 0.01 e V_{0} \lesssim T_{S} \lesssim 0.1 e V_{0} \\ V_{0}^{3}, & T_{S}>0.1 e V_{0}\end{cases}
$$

where we use $V_{0}$, which is connected with the density of electrons at the electrosphere (see eq. [1]).

For the most conservative parameters of SQM $\left(m_{s} \simeq 150 \mathrm{MeV}\right.$ and $\mu \simeq 300 \mathrm{MeV}$ ), the value $V_{0}$ increase $\sim 3$ times because of the surface effects. Hence, at high temperatures $L_{ \pm}$may increase up to a few $\times 10$ times in comparison with the value calculated by Usov (1998) for the electrosphere model in which the surface effects are ignored. 
It has been shown by Cheng \& Harko (2003) that quarkquark bremsstrahlung (and another radiation from SQM) may be strongly suppressed in the process of its propagation through the electrosphere. Most probably, the increase of the density of electrons by a factor of $\sim 20-30$ because of the surface effects results in the almost complete suppression of the outgoing radiation from SQM if the surface temperature is not very high $\left(T_{S}<1 \mathrm{MeV}\right)$. In this case, practically all the radiation of a bare strange star is generated by the electron layer at $z>0$. In contrast, we expect that the electron density increase does not significantly affect the electron-electron bremsstrahlung radiation from the electrosphere at rather low temperatures $\left(T_{S}<\right.$ $0.1 \mathrm{MeV}$ ), where this radiation prevails. Indeed, in this case the bremsstrahlung radiation is mainly generated at the distance from the SQM surface at which the local plasma frequency of electrons is equal to the frequency of radiation (Jaikumar et al. 2004). Since at any temperature the plasma frequency of electrons is directly connected with the density of electrons, the density of electrons in the radiating region is more or less the same irrespective of the structure of the electron layer. Therefore, the electron-electron bremsstrahlung radiation at a given frequency is more or less the same, too. We hope to deal with detail calculations of the thermal emission from a bare strange star for the electrosphere model developed in this paper elsewhere.

Recently, the cooling of young bare strange stars has been studied numerically using the electrosphere model where the surface depletion of $s$ quarks is ignored (Page \& Usov 2002). It was shown that the thermal luminosity of such a star in photons and $e^{+} e^{-}$pairs may be up to $\sim 10^{49}-10^{50} \mathrm{ergs} \mathrm{s}^{-1}$ for a few seconds after the star formation and remains high enough $\left(\gtrsim 10^{36} \mathrm{ergs} \mathrm{s}^{-1}\right)$ as long as the surface temperature is higher than $\sim 0.1 \mathrm{MeV}$. The increase of the density of electrons in the electrosphere because of the surface effects has to modify the light curves calculated by Page \& Usov (2002). We expect that at the first stage of the strange star cooling, when neutrino losses dominate, the thermal radiation from the stellar surface increases in accordance with equation (38), i.e., by a factor of few times 10 at $T_{S}>0.1 e V_{0}$. At the second stage, when the losses in the surface thermal radiation prevails, the thermal luminosity decreases more rapidly than in the case of the nonmodified electrosphere, especially at low temperatures $\left(T_{S}<0.1 \mathrm{MeV}\right)$ where the main mechanism of the thermal emission is the electron-electron bremsstrahlung that is ignored by Page \& Usov (2002).

At the surface of a strange star a massive normal matter crust may be formed by accretion of matter onto the star. From Figure 3 we can see that at rather low temperatures $\left(T_{S} \ll\right.$ $\left.T_{*} \sim 10 \mathrm{MeV}\right)$ the electrostatic potential of electrons $e V_{0}$ is more than the electron chemical potential $(\sim 25 \mathrm{MeV})$ at which neutron drip occurs (Baym et al. 1971). Therefore, the maximum density of the crust is limited by neutron drip and is $\sim 4.3 \times$ $10^{11} \mathrm{~g} \mathrm{~cm}^{-3}$ (Alcock et al. 1986). In this case, the maximum mass of the crust is $\sim 10^{-5} M_{\odot}$.

This work was supported by a RGC grant of the Hong Kong Government. V. V. U. would like to thank the Department of Physics, University of Hong Kong, where this work in part was carried out, for its kind hospitality. The research of V. V. U. was supported by the Israel Science Foundation of the Israel Academy of Sciences and Humanities.

\section{REFERENCES}

Aksenov, A. G., Milgrom, M., \& Usov, V. V. 2003, MNRAS, 343, L69

Alcock, C., Farhi, E., \& Olinto, A. 1986, ApJ, 310, 261 2004, ApJ, 609, 363

Alford, M. 2001, Annu. Rev. Nucl. Part. Sci., 51, 131

Alford, M., Bowers, J., \& Rajagopal, K. 2001a, J. Phys. G, 27, 541 2001b, Phys. Rev. D, 63, 074016

Alford, M., Kouvaris, C., \& Rajagopal, K. 2004, Phys. Rev. Lett., 92, 222001

Alford, M., \& Rajagopal, K. 2002, J. High Energy Phys., 6, 031

Alford, M., Rajagopal, K., \& Wilczek, F. 1998, Phys. Lett. B, 422, 247

Alford, M., \& Reddy, S. 2003, Phys. Rev. D, 67, 074024

Bailin, D., \& Love, A. 1984, Phys. Rep., 107, 325

Baym, G., Pethick, C. J., \& Sutherland, P. G. 1971, ApJ, 170, 299

Berger, M. S. 1991, Phys. Rev. C, 44, 566

Berger, M. S., \& Jaffe, R. L. 1987, Phys. Rev. C, 35, 213

Bodmer, A. R. 1971, Phys. Rev. D., 4, 1601

Bowers, J. A., \& Rajagopal, K. 2002, Phys. Rev. D, 66, 065002

Cheng, K. S., Dai, Z. G., \& Lu, T. 1998, Int. J. Mod. Phys. D, 7, 139

Cheng, K. S., \& Harko, T. 2000, Phys. Rev. D, 62, 083001

. 2003, ApJ, 596, 451

Evans, N., Hormuzdiar, J., Hsu, S. D. H., \& Schwetz, M. 2000, Nucl. Phys. B, 581,391

Farhi, E., \& Jaffe, R. L. 1984, Phys. Rev. D, 30, 2379

Glendenning, N. K. 1996, Compact Stars: Nuclear Physics, Particle Physics, and General Relativity (New York: Springer)
Haensel, P., Zdunik, J. L., \& Schaeffer, R. 1986, A\&A, 160, 121

Hu, J., \& Xu, R. X. 2002, A\&A, 387, 710

Huang, M., \& Shovkovy, I. 2003, Nucl. Phys. A, 729, 835

Jaikumar, P., Gale, C., Page, D., \& Prakash, M. 2004, Phys. Rev. D, 70, 023004

Kettner, Ch., Weber, F., Weigel, M. K., \& Glendenning, N. K. 1995, Phys. Rev. D, 51, 1440

Lugones, G., \& Horvath, J. E. 2003, A\&A, 403, 173

Madsen, J. 2000, Phys. Rev. Lett., 85, 4687 2001, Phys. Rev. Lett., 87, 172003

Page, D., \& Usov, V. V. 2002, Phys. Rev. Lett., 89, 131101

Rajagopal, K., \& Wilczek, F. 2001, Phys. Rev. Lett., 86, 3492

Rapp, R., Schäfer, T., Shuryak, E. V., \& Velkovsky, M. 1998, Phys. Rev. Lett., 81,53

Schäfer, T. 2000, Nucl. Phys. B, 575, 269

Schwinger, J. 1951, Phys. Rev., 82, 664

Shovkovy, I., \& Huang, M. 2003, Phys. Lett. B, 564, 205

Steiner, A. W., Reddy, S., \& Prakash, M. 2002, Phys. Rev. D, 66, 094007

Usov, V. V. 1998, Phys. Rev. Lett., 80, 230

2001a, ApJ, 550, L179

2001b, Phys. Rev. Lett., 87, 021101

2004, Phys. Rev. D, 70, 067301

Weber, F. 1999, J. Phys. G, 25, 195

2004, Prog. Part. Nucl. Phys., 54, 193

Witten, E. 1984, Phys. Rev. D, 30, 272 